\title{
Ethical challenges experienced by public health nurses related to adolescents' use of visual technologies
}

\section{Authors}

Hilde Laholt ${ }^{1}$, Kim McLeod ${ }^{2}$, Marilys Guillemin ${ }^{3}$, Ellinor Beddari ${ }^{1}$, Geir Lorem ${ }^{1}$

\section{Institutions}

${ }^{1}$ UiT Norwegian Arctic University, ${ }^{2}$ University of Tasmania, Australia. ${ }^{3}$ University of Melbourne, Australia

Corresponding Author: Hilde Laholt: hilde.laholt@uit.no

Short Title: "Ethical challenges in school nursing."

Key words: Public health nurses, ethical challenges, school nursing, adolescents, visual technologies 


\begin{abstract}
Background: Visual technologies are central to youth culture, and are often the preferred communication means of adolescents. Although these tools can be beneficial in fostering relations, adolescents' use of visual technologies and social media also raises ethical concerns.
\end{abstract}

Aims: We explored how school public health nurses identify and resolve the ethical challenges involved in the use of visual technologies in health dialogues with adolescents. Research Design: This is a qualitative study utilizing data from focus group discussions. Participants and research context: We conducted focus group discussions using two semistructured discussion guides with seven groups of public health nurses $(n=40)$ working in Norwegian school health services. The data were collected during January and October 2016. Discussions were audio recorded, transcribed and coded into themes and subthemes using systematic text condensation.

Ethical considerations: The leader of the public health nursing services and the Norwegian Centre for Research Data approved the project. All participants gave informed consent. Findings: In adolescents' use of visual materials with public health nurses, ethical concerns were raised regarding suicide ideations, socially unacceptable content, violation of privacy, and presentations of possible child neglect. The nurses utilized their professional knowledge and experience when identifying and navigating these ethical dilemmas; they resolved ethical uncertainties through peer discussion and collaboration with fellow nurses and other professionals.

Discussion: We discussed the findings in light of Annemarie Mol's interpretation of the ethics of care. Mol expands the notion of ethical care to include the action of technologies. Conclusion: Although the increasing use of visual technologies offered benefits, school 
nurses faced ethical challenges in health dialogues with adolescents. To address and navigate these ethical issues, they relied on their experience and caring practices, based on their professional ethics. Uncertainties were resolved through peer dialogue and guidance.

\section{Introduction}

Visual technologies such as smartphones and other mobile phones are part of western youth culture $^{1,2}$. These visual technologies are increasingly becoming a feature of professional interactions between public health nurses (PHNs) and adolescents. These technologies can be beneficial in shaping relations and enabling communication ${ }^{3}$. However, there are challenges associated with the increasing use of visual technologies in schools.

PHNs in Norway have a key role in health promotion and illness prevention ${ }^{4}$. PHNs regularly meet with young people (6-20 years) from primary through high school through 'health dialogues' in schools. This places PHNs in a unique position to identify and address challenges faced by adolescents. The 'health dialogue' is a dialogue between nurses and pupils with the aim of promoting health and preventing disease by raising awareness and addressing health-related behaviours ${ }^{4,5}$. We have previously reported how PHNs in northern Norway perceive their professional practice as a relational practice, and use a variety of visualization methods as part of their health dialogue with pupils; we have shown that PHNs are aware of the benefits and challenges of using visual methods in school nursing ${ }^{3}$. PHNs working in schools focus on providing care based on respect for each individual's situation; this care is based on relationships and shared work involving the pupils, their families, teachers and other professionals ${ }^{6}$. Norwegian PHNs abide by the ethical guidelines of the Norwegian Nurses' Organisation (NNO); the Child Welfare Act underlines that in health care 
services all considerations shall be in the best interest of the child ${ }^{7-9}$.

The use of visual technologies is a relatively new field for PHNs. Adolescents use their mobile phones for communication, and to support and enhance their social relations ${ }^{2,10}$. We therefore wanted to explore the ethical challenges PHNs encountered due to the increasing use of visual technologies by young people, as well as how PHNs navigated and resolved those challenges. We focused on both the visual materials and the young people involved in the health dialogue. Annemarie Mol's interpretation of the ethics of care is useful to understand the manner in which PHNs navigate ethical issues ${ }^{11}$. We have shown elsewhere that PHNs have a flexible, relational practice ${ }^{3}$. Mol expands the notion of ethical care to include the action of technologies ${ }^{11}$; and her notion of the "ethics of care" enables an understanding of how PHNs navigate ethical issues as part of their ongoing practice. Mol argues that it is not productive to apply external general principles of good practice, and then judge the situation according to these. In biomedical ethics, respect for patients' autonomous choices is fundamental, and to arrive at the best ethical decision, ethical principles are weighed up, maximizing good choices and consequences, and minimizing bad ones ${ }^{12,13}$. In contrast to the biomedical approach to ethics, Mol proposes that solutions to problems are formulated in local practices, stating that ethical work involves attending carefully to what happens in practice, reflecting on the outcome, and making adjustments ${ }^{11}$. The focus is on the broader process in which the patient or user of health care services is embedded, which can include technology, habits, skill, propensities and hopes. This interpretation of ethics is not established in opposition to traditional biomedical ethics. Ethical norms like justice are still involved, not as a basic principle as biomedical ethics suggests, but as one of the elements that can be brought to bear in a situation ${ }^{11,12}$. In this instance, the ethical terrain expands to include how PHNs respond to all components of the encounter, including the effect of the 
visual materials shared by the young people. Using Mol's conception of ethical care, we argue that when faced with ethical challenges in the use of visual technologies, PHNs employed their professional experience and expertise. To address ethical challenges arising from adolescents' use of visual technologies, PHNs relied on common ethical values, their professional knowledge, past experience from similar situations, peer dialogue and guidance from other PHNs and professionals.

\section{Aim}

We explored how PHNs identify and resolve ethical challenges involved in the use of visual technologies in health dialogues in schools.

\section{Design and method}

This was a qualitative study utilizing focus group discussions (FGDs) with PHNs to explore our research aim ${ }^{14,15}$. This article is a part of a larger study on how PHNs understand their role, and how they use visual methods in school nursing ${ }^{3}$. The project took place in Troms $\emptyset$, a town of approximately 73000 inhabitants in northern Norway. The Norwegian Centre for Research Data approved the study on 8 September 2015 (NSD: Ref. No. 4439), and the leader of the public health nursing services approved the project. The first author (HL) attended a meeting with almost all the PHNs working in Troms $\varnothing$ (about 60), informed them about the project and invited them to participate. The consent process was explained, emphasising that the attendees were under no obligation to participate. All participants provided written consent. 


\section{Participants}

A total of 40 PHNs agreed to participate in the project in seven focus groups. Thirty-one PHNs joined FGDs 1 to 5 in five different health clinics in January and February 2016 (phase one), and nine PHNs participated in FGDs 6 and 7 in September and October 2016 (phase two), at UiT the Arctic University of Norway. Eight of nine PHNs were re-interviewed in phase two (Table 1). The PHNs in Troms $\varnothing$ were all female and registered nurses with further education as PHNs. Most of them worked in health clinics (for children aged 0-5 years) and school health services (for pupils aged 6-20 years). Their work experience ranged from four months to 34 years in school nursing. Since the principal researcher and FGD moderators are PHNs, we considered the impact of the relationship between the researchers and participants. However, the focus group design allowed access to descriptions where the relationship was of little relevance; the participants discussed actively, and were able to bring up any ethical issues concerning the study ${ }^{15}$.

Table 1 Characteristics of focus groups and the participants

\begin{tabular}{lll}
\hline $\begin{array}{l}\text { Focus group } \\
\text { (in date order) }\end{array}$ & $\begin{array}{l}\text { No. of } \\
\text { attendees }\end{array}$ & $\begin{array}{l}\text { Experience range } \\
\text { in years }\end{array}$ \\
\hline 1 & 5 & $0.4-9$ \\
2 & 5 & $0.6-34$ \\
3 & 11 & $1-21$ \\
4 & 6 & $2-17$ \\
5 & 4 & $8-17$ \\
6 & 4 & $1-30$ \\
7 & 5 & $3-23$ \\
Total & 40 & \\
\hline
\end{tabular}




\section{Data collection}

We conducted seven FGDs (Table 1). FGDs 1-5 were conducted prior to a professional development programme in visual methods for the PHNs, and FGDs 6 and 7 followed the programme. As part of the development programme the participants used different visual methods such as drawing, images, films, and photo elicitations in their health dialogues with school pupils ${ }^{16-18}$. Through workshops, PHNs reported back on their experiences of using visualization in the school health dialogue. We started the FGDs with an introduction and explained the aim of the focus groups. In FGDs 1-5, we used a semi-structured discussion guide to explore ethical issues related to the health dialogue in schools, and the use of visual methods and social media in school nursing. We used a different interview guide in FGDs 5 and 6; here we asked the PHNs to discuss their experiences and ethical challenges after they had participated in the development programme in visual methods and had observed visual methods in their practice. HL was the moderator for all FGDs, with one co-moderator present in FGDs 1-5 (REO) ${ }^{3}$, and another co-moderator in FGDs 6 and 7 (EB). We allowed the participants to reflect and their ideas were allowed to emerge and be discussed ${ }^{14,15}$. The FGDs lasted from 73-91 minutes, and were recorded with a digital voice recorder. After the FGDs, the moderator and co-moderator discussed the process and provided written reflections.

\section{Data analysis}

HL transcribed the audio recordings verbatim from the FGDs. The transcripts were analysed using systematic text condensation ${ }^{19}$, and we used NVivo 11 to systematize the material ${ }^{20}$. Systematic text condensation draws on Giorgi’s psychological phenomenological analysis and 
consist of four steps. In the first step, HL read the 111 pages of transcripts to get a general impression of the whole material. At this stage, we looked for preliminary themes associated with how PHNs identify and resolve ethical challenges involved in the use of visual technologies in health dialogues in schools. We tried to remain atheoretical while admitting an interpretative position determined by research question ${ }^{19}$. In step 2 , HL used Nvivo 11 to organize the material ${ }^{20}$. The transcripts were systematically reviewed line by line to identify meaning units and themes. We identified and coded the themes and the coded data were condensed and abstracted within each of the categories. The research group collaborated in steps 3 and 4. In step 3, we reduced the empirical data to a decontextualized selection of meaning units sorted as thematic code groups. In step 4, we reconceptualized the data and put the pieces together again. The analysis resulted in three key findings presented here.

\section{Findings}

The first reading revealed eight themes that forms the total impression of our material: school nursing and use of visualization, relational aspects, challenges with social media, the user perspective, young people and visualization, ethical challenges in the use of visual technologies, closeness and distance, and public health and visualization. The final descriptions were a result of a process moving back and forth between the transcripts, the findings and theoretical perspectives. We present the findings as: situations that raised ethical issues, identifying and navigating ethical challenges and resolving the ethical challenges through peer dialogue.

We found that PHNs experienced certain situations in which the use of visual materials raised ethical challenges. Although they were accustomed to experiencing ethical issues in their 
health dialogues with adolescents, the use of visual technologies created different kinds of ethical challenges. Visual presentations are a more direct form of communication, having the capacity of touching people's feelings, and eliciting strong bodily and sensory responses. This was especially evident in the visual materials generated by adolescents that indicated suicide ideation, were socially unacceptable, violated privacy, or were presentations of possible child neglect. PHNs processed these challenging situations by drawing on their professional expertise, practical experience, and professional ethical values. Ethical uncertainties were resolved through dialogue with peer PHNs and other professionals.

\section{Situations that raised ethical issues}

Although the issues raised by adolescents in the health dialogue with PHNs were familiar, the adolescents' use of visual tools presented new types of challenges. In the FGDs, the PHNs discussed typical challenging situations arising from the use of the visual technologies. These included adolescents in mental distress presenting or texting pictures of suicide ideations. One PHN talked about a situation in which a teacher needed help when a girl had sent an image from her smartphone showing that she wanted to kill herself. Communication through a smartphone was experienced by the PHN as direct, and yet distant. The situation was thus perceived as more ethically challenging than an ordinary face to face conversation. Although the suicide ideation was taken seriously by the PHN, the PHN did not definitively know from the content of the image whether the pupil actually wanted to kill herself. It could have been a call for help with her problems or an expression of desperation that required immediate attention. Based on their professional knowledge and experience, PHNs know that such situations represent concern for an adolescent's health and wellbeing. For the PHN it became important to explore what this young person wanted to achieve with her communication. 
PHNs are trained in caring for young people in mental distress, and this PHN decided to meet the girl face to face. The PHN could then ask specific questions, exploring any potential risk of suicide, and provide care for her.

Another example discussed by the PHNs in the FGDs was when a pupil came on his own initiative and showed them a film with inappropriate content. The PHN said: "Once an adolescent came to my office, sharing a film he had taken at home of his drunken stepfather. That was challenging, especially because he thought the film was funny." The PHN was concerned about the film and its content, and by the reaction from this adolescent. Watching a video is a more direct form of communication than listening to a verbal story from an adolescent about a drunken stepfather. This example presented a number of challenges, including a challenge to the PHN's professional objectivity with the possibility of becoming overinvolved in this young person's situation. The PHN reflected on the reasons this adolescent showed her the video. Did he intend to alert her to his difficult home situation, and what did he mean by his laughing? Was he considering this film as funny because his stepfather did stupid things on a video, or was the teenager's laughter a response to being uncomfortable when meeting a PHN? The PHN found this situation problematic, and this meeting "became a starting point for further work". In this situation it was important for the PHN to explore the adolescent's' intentions in coming to see her, and showing this video; this was necessary to determine the required steps to assist the boy. In the FGD, the PHNs discussed the ethical challenges in visualization presented by adolescents. When faced with these ethical concerns, the PHNs used their professional knowledge and past experience to decide how to proceed. 


\section{Identifying and navigating ethical challenges}

When PHNs became aware of ethical concerns in their visual interactions with adolescents, they also drew on common ethical principles, such as autonomy and maximizing life and health. In the example of the girl's suicide ideation, the PHN was touched and emotionally affected by the content and the way the message was presented. The PHN realized that this communicative act represented a potential ethical dilemma where an adolescent's life could be at stake. The PHN used her experience in dealing with young people in mental distress, and decided to call this adolescent, offering a health dialogue at the school nursing office. After several health dialogues, the PHN explained to the adolescent that she had to inform the girl's parents and other health professionals about the situation, even if this girl did not agree. The PHN said: "She was not particularly happy about me when she left the school nursing office, and she never came back to my office again”. Building and enabling relationships are fundamental in PHNs' practice. However, the PHN believed that the ethical dilemma of life and death justified the breach of confidentiality, even though this damaged the relationship between the PHN and the adolescent.

Verbal presentations of turbulent family life were a familiar issue for PHNs working in schools. However, visual presentations such as that of the drunken stepfather are more direct, thus triggering an immediate reaction. This situation presented compound issues. The PHNs discussed why adolescents chose to come on their own initiative and show them visual materials in their health dialogues. One experienced PHN said, "I think that pupils are coming and showing us sensitive images and films because they want a professional adult's view and 
help on their difficult life situations". PHNs considered it as a vote of confidence when young people invited them into their lives. In the example of the drunken stepfather, the PHN became concerned and uncertain about the reasons why the young person came and presented this film. This situation was experienced as ethically challenging because it raised questions about the family situation and the child's wellbeing; it was important to ask sensitive and clear questions to get an insight into this person's background and family relations. This PHN relied on her past experience from other situations of possible child neglect when deciding whether this video represented a fraught family situation, or a one-time episode where a drunken adult did funny things in a video. She also had to consider challenges in filming a drunken adult without his knowledge, and ethical issues connected to contacting and communicating with the parents if or when this became necessary. The PHNs also considered challenges in how the adolescent's parents would react when they realized that their son had shown her this kind of video. The parents could feel ashamed and become angry with the PHN and refuse to meet her, because she had gained visual insight into their private life. The weighing up of not harming the relationship between a young person and his parents, as against potential damage caused by possible child neglect, made this situation an ethical dilemma for the PHN.

Visual technologies in health dialogues provided new ethical issues for the PHNs, who therefore wanted to be prepared before using such tools in school nursing. The PHNs discussed situations of ethical concern when pupils distributed visual material that was socially unacceptable, or violated pupils' privacy. The nurses communicated their fear of losing control if adolescents wanted to use social media in a way that could potentially harm other adolescents. One experienced PHN expressed uncertainty about utilizing Snapchat as a communication method in health dialogues. She said: "If I'm having group sessions about 
puberty and sexuality and a boy sends images of sensitive parts of himself, and suddenly the Snap is screenshotted and then it's gone, how can I handle that?" One PHN stated her standpoint when introducing new tasks in group health dialogues saying: "If we give adolescents tasks, we have to communicate what we want because we know that some adolescents will step over the line." PHNs knew from experience from similar situations that some adolescents test limits of right and wrong in their interactions. Other adolescents want to joke, or bully, or harm other pupils. Some adolescents want to present an image to get attention or provoke a reaction from adults, or to be funny at the expense of others. However, the PHNs conveyed their duty to protect pupils from doing things they would later regret, and to protect them from harming themselves and other pupils. PHNs underlined their responsibilities in practical and ethical issues when introducing visual technologies and social media in health dialogues, especially ethical issues involving data security. They were thus aware of ethical issues in their work and wanted to be prepared to face them.

\section{Resolving the ethical challenges through peer dialogue}

Although PHNs relied on their expertise and experience to address ethical challenges with visual technologies, sometimes uncertainty or indecision remained. PHNs processed these uncertainties as part of peer collaboration (e.g. meetings, discussions, guidance). PHNs reflected, discussed, made adjustments and navigated ethical issues as part of their work in schools. When adolescents posted unacceptable images on social media, or sent nasty messages to each other, the PHNs expressed their uneasiness about how to handle such situations. One PHN said: "Adolescents are coming to me and presenting bullying and harassing messages from other pupils, and I wonder how to respond”. When PHNs were uncertain about how to proceed, they sought peer dialogue and guidance from other PHNs, 
health professionals or social workers. PHNs working in school health services are used to working alone a great deal, and they have to make decisions on their own. One experienced PHN described their role in secondary school: "Most of the time we're the only health care workers in the school system". When faced with new ethical issues and feeling uncertain about how to handle such situations, the PHNs called more experienced colleagues working at other schools. They freely contacted other PHNs when they needed help. One PHN said: “PHNs see many 'normal pupils', so if I meet one I'm concerned about, I need help. Then I have to seek advice and guidance from other professionals, sometimes child welfare". The PHNs reported having access to peer group guidance with other PHNs, regularly discussing practical and ethical issues. There were also groups led by a child psychologist or a social worker. Peer discussions and guidance provided support or challenged the choices PHNs make, enabling them to reflect on their own practice from an outsider perspective, and to see themselves from the other's point of view.

\section{Discussion}

PHNs commonly deal with complex problems and ethical issues in their work. Our study showed how PHNs became aware of new ethical issues arising from adolescents' use of visual technologies, and how they addressed and navigated these ethical challenges. When PHNs were uncertain how to resolve these challenges, they contacted colleagues or other professionals for peer dialogue and guidance. As a result, they expanded their professional ethical practice to include the new types of challenges presented by visual technologies, and in doing so, undertook what Mol ${ }^{11}$ describes as ethical work.

PHNs reported challenges connected to adolescents' use of visual technologies in health 
dialogues. Visual presentations are a more symbolic and direct form of communication, with the capacity to elicit bodily and sensory responses and activate memories ${ }^{21,22}$. For example, when a young person presented suicide ideations through a smartphone, the PHN was alerted to an ethically important situation. The PHNs in our study were attentive to all components of the encounter, including the communication presented by the visual tool ${ }^{11}$. PHNs knew from professional experience that all suicide ideations should be taken seriously. However, they were also aware that teenagers are in a development phase where some react to difficulties with strong emotions, and others want to provoke or shock through their communication ${ }^{23}$. Clancy and Svensson ${ }^{24}$ described how PHNs' responsibilities for the other cannot be ignored, avoided or transferred. This commitment extends to the ethical problems provoked by visual materials, described by one PHN as a "starting point for further work". The PHNs in our study showed that they orientated to the overall 'good' of improving the young person's wellbeing ${ }^{11}$. PHNs were familiar with verbal presentations of turbulent family relationships. However, the video presentation featuring a drunken step-father was more direct and triggered a more emotive response in the PHN concerned ${ }^{22}$. She described her discomfort when faced with the potential ethical issue. There was no clear "right" way to proceed. However, Mol's perspective allows us to approach right and wrong as entwined, and as sometimes complex and ambivalent ${ }^{11}$. Some teenagers present a video to get attention, and to shock or provoke a reaction from an adult. However, adolescents need to be met with respect and understanding. The PHN had to communicate sensitively to ensure the adolescents' health and wellbeing, but at the same time considered possible child neglect ${ }^{9}$. Care is an interactive, open-ended process where PHNs know that some adolescents need help and want an adult's view of their problems, while others want a dialogue about the challenges life presents to a teenager ${ }^{11,23}$. However, PHNs could never be completely certain that their ethical decision was correct. When facing challenging situations, they proceeded with caution, and sought other colleagues 
for discussion and guidance. Our findings show that they always had to be prepared to be flexible and expanded their practice to solve ethical issues that arose in new types of situations. The PHNs showed a willingness to engage in a variety of efforts to try to improve the situation of the young person, which Mol characterizes as good care: this also described how new ethical situations are incorporated in their clinical/practical repertoire when relating to adolescents or young adults ${ }^{11}$.

The orientation to the overall 'good' of improving a young person's wellbeing also showed how PHNs engaged with ethical norms in their practice. This approach is exemplified by the PHN who broke a promise of confidentiality with a pupil, due to her concern for the adolescent's health and wellbeing. The PHN had ongoing regard for maintaining confidentiality with the pupil, but had to modify how she applied this as the situation with the pupil evolved. Building and enabling relationships are a fundamental part of the practice of PHNs. However, the PHN's decision on confidentiality was influenced by what the visual material contributed to the situation ${ }^{11}$. The ethical issue of life and death justified the breach of confidentiality and the ensuing damaged relationship ${ }^{12}$. This indicates how PHNs make ongoing adjustments in their decision making, based on contextual elements ${ }^{11}$. Here, confidentiality is not a foundational principle to be applied in a static way from outside the situation, but as an approach which can inform how a situation is navigated. This illustrates that PHNs do not adhere to one pre-determined 'good' outcome for the pupil. Instead, they are aware that in their efforts to improve the overall wellbeing of a young person, multiple 'goods' co-exist, so they work ethically to find balances and compromises between the various goods 25 . 
PHNs also expressed their understanding of how to guide young people in relation to the ethical issues that arise with visual technologies and social media. PHNs showed ethical awareness in communicating their responsibilities for practical and ethical issues connected to data security before introducing visual technologies and social media in their school health dialogues. Most adolescents are aware that they are not allowed to physically harm, or verbally harass each other face to face. However, rules on social media are less clear and adolescents are testing the limits of right and wrong in these social arenas ${ }^{10}$. In Norway, 55\% of adolescents aged 15-16 years had sent or posted online images they regretted through the internet ${ }^{10}$. The PHNs underlined their duty to protect teenagers from causing harm to themselves or other vulnerable people. They knew from experience that some adolescents are capable of misusing visual technologies by spreading sensitive material through social media. The PHNs took up opportunities of promoting health and changing bad behavior in groups of adolescents, guiding them about the use of smartphones and the internet ${ }^{4,5}$. The role of health promotion in school nursing is to activate resources and provide appropriate skills for young people to deal with life's challenges ${ }^{26}$. Olweus emphasizes the importance of teaching pupils, parents and teachers about visual technologies, to ensure safe and ethical behavior ${ }^{27}$.

For Mol ${ }^{11}$, to contextually achieve 'good', activities must be attuned to the young person's needs, and this involves coordination between all those involved. The PHNs were mindful of the broader context the adolescents were embedded in, and considered various activities that would provide a good outcome on an individual basis. They also engaged with others involved in the young people's care and education. The team was involved in discussions about the wellbeing of adolescents ${ }^{11}$, and PHNs actively collaborate with other professionals 28. When faced with ethical uncertainties, the PHNs in our study highlighted contacting other PHNs for dialogue and guidance. Mol ${ }^{11}$ helps us to see this not as a weakness, but rather as a 
way of trying new approaches, to experiment and modify one's practice. In this way, the PHNs were expanding their relational, flexible practice to include new ethical problems ${ }^{3}$.

\section{Strengths and limitations}

The study had both strengths and limitations relating to the sample, and the fact that the moderators are PHNs, as well as the use of focus groups as a method. Regarding the sample, there was a high level of engagement and participation of PHNs in Troms $\varnothing$. We recruited the participants through the leader of the public health nursing services, and conducted the study on Fridays, normally meeting days for the PHNs. Taking over existing meeting days made it easier to ensure attendance. Most nurses who chose to take part in this study were likely to have a strong interest in developing their professional practice. However, we do not know if there were critical PHNs who chose not to participate. HL and the co-moderators in FGDs 1-5 $(\mathrm{REO})^{3}$ and 6-7 (EB) were "insiders", and knew several of the participants through their role as teachers on the PHN master's course. This insider status may have influenced the recruiting process and engagement in the FGDs. With regard to the use of FGDs, individual interviews could be perceived as more suitable than FGDs for exploring ethical issues ${ }^{15}$. However, our participants were familiar with discussing ethical issues from peer group discussions. In our FGDs, they wanted to share their experiences and discuss the challenging practice issues they faced. The group dynamics allowed them to reflect, and discuss their experiences with their peers ${ }^{15}$. We used systematic text condensation for our analysis in accordance with the aim of the study ${ }^{19}$. HL interpreted the data, and then the research group consisting of two PHNs, one philosopher, and two health sociologists re-analysed and discussed the findings, until agreement was reached. The systematic steps of the analytic method and the interpretative discussion of the research group provided a more nuanced analysis which supported the 
validity of the findings, and helped to ensure reliability ${ }^{29,30}$. Our findings contribute to knowledge of the professional practices of PHNs working in schools, and may be transferable to similar settings in which nurses and other social and health workers are involved with young people.

\section{Conclusion}

Visual technologies can be beneficial in relationship building and communication strategies, but also represent new kinds of ethical challenges in health dialogues. To address and navigate these ethical challenges, PHNs relied on their professional knowledge, ethical awareness and past experiences, which were based on their existing professional sense of ethics. Ethical uncertainties were resolved as part of peer dialogue and guidance.

\section{Acknowledgements}

We would like to thank the study participants for their contributions and Randi Elisabeth Olsen for assistance in planning and conducting the study.

\section{Declaration of Conflicting interests}

The author(s) declared no potential conflicts of interest with respect to the research, authorship, and/or publication of this article. 


\section{References}

1. Pink S. The visual in ethnography: Photography, video, cultures and individuals. SAGE Visual Methods 2012: 123.

2. Vanden Abeele MM. Mobile lifestyles: Conceptualizing heterogeneity in mobile youth culture. New Media \& Society 2016; 18: 908-926. DOI: 10.1177/1461444814551349.

3. Laholt, H Guillemin M, McLeod K, Lorem. Visual methods in health dialogues: A qualitative study of public health nurse practice in schools. J Adv Nurs 2017; 73 (12): 30703078. DOI: 10.1111/jan.13371.

4. Norwegian Directorate of Health. Nasjonale retningslinjer for helsestasjon (0-5 år) skolehelsetjenesten (6-20 ̊̊r) [National guidelines for health clinics (0-5 years) and school health services (6-20 years], https://helsedirektoratet.no/retningslinjer/helsestasjons-ogskolehelsetjenesten (2017).

5. Borup I. The school health nurse's assessment of a successful health dialogue. Health Soc Care Community 2002; 10: 10-19.

6. Ivanov LL and Oden TL. Public health nursing, ethics and human rights. Public Health Nurs 2013; 30: 231-238. DOI: 10.1111/phn.12022.

7. International Council of Nurses. NewsCAP: The International Council of Nurses (ICN) updates its code of ethics. Am J Nurs 2013; 113: 15. DOI:

10.1097/01.NAJ.0000427863.90355.22.

8. Norsk Sykepleierforbund. Yrkesetiske retningslinjer for sykepleiere ICNs etiske regler [The ethical guidelines of the Norwegian Nurses Organisation (NNO)]. Oslo: Norsk Sykepleierforbund, 2011.

9. Lov om barneverntjenester (barnevernloven) [Act relating to Child Welfare Services] (Child Welfare Act) Changed 01.01.2016. Norway: Ministry of Children and Equality, 1992. 
10. Medietilsynet. Children and media 2016 - Facts about children and youths`(9-16) use and experience of media. 2016. Fredrikstad: Medietilsynet.

11. Mol A. The logic of care: Health and the problem of patient choice. London:

Routledge, 2008.

12. Beauchamp TL and Childress JF. Principles of biomedical ethics. 7th ed. New York: Oxford University Press, 2013.

13. Veatch RM. The basics of bioethics. London: Routledge, 2016.

14. Krueger RA and Casey MA. Focus groups: A practical guide for applied research. Thousand Oaks, CA: Sage publications, 2014.

15. Barbour R. Doing focus groups. London: Sage, 2007.

16. Guillemin M. Understanding illness: Using drawings as a research method. Qual Health Res 2004; 14: 272-289. DOI: 10.1177/1049732303260445.

17. McLeod K. Wellbeing Machine: How health emerges from the assemblages of everyday life. United States ISBN 978-1-61163-705-2: Carolina Academic Press, 2017.

18. Rose G. Visual methodologies: an introduction to researching with visual materials. 3rd ed. Los Angeles: Sage, 2012.

19. Malterud K. Systematic text condensation: a strategy for qualitative analysis. Scand $J$ Public Health 2012; 40: 795-805.

20. QSR International Pty Ltd. NVivo qualitative data analysis software; QSR International Pty Ltd. Version 11. 2015.

21. McLeod K and Guillemin M. The Impact of Photographs on the Researcher: An Ethical Matter for Visual Research. Ethics and Visual Research Methods Theory, Methodology and Practice. Springer, 2016, p. 89-100.

22. Mitchell C. Doing visual research. London: Sage, 2011. 
23. von Tetzchner S. Utviklingspsykologi [Development psychology]. 2nd ed. Oslo: Gyldendal akademisk, 2012.

24. Clancy A and Svensson T. 'Faced' with responsibility: Levinasian ethics and the challenges of responsibility in Norwegian public health nursing. Nurs Philos 2007; 8: 158166. 2007/06/22. DOI: 10.1111/j.1466-769X.2007.00311.x.

25. Mol A, Moser I, Piras EM, et al. Care in Practice. On Normativity, Concepts, and Boundaries. 2011.

26. Olsson CA. Adolescent resilience: A concept analysis. J Adolesc 2003; 26: 1.

27. Olweus D. School bullying: Development and some important challenges. Anпи Rev Clin Psychol 2013; 9: 751-780.

28. Clancy A, Gressnes T and Svensson T. Public health nursing and interprofessional collaboration in Norwegian municipalities: a questionnaire study. Scand J Caring Sci 2013; 27: 659-668.

29. Malterud K. Qualitative research: standards, challenges, and guidelines. Lancet 2001; 358: 483-488. 2001/08/22. DOI: 10.1016/s0140-6736(01)05627-6.

30. Tong A, Sainsbury P and Craig J. Consolidated criteria for reporting qualitative research (COREQ): a 32-item checklist for interviews and focus groups. Int J Qual Health Care 2007; 19: 349-357. DOI: 10.1093/intqhe/mzm042. 